\title{
HLA-DR Antigen Type
}

National Cancer Institute

\section{Source}

National Cancer Institute. HLA-DR Antigen Type. NCI Thesaurus. Code C128962.

The determination of the amount of human leukocyte antigen, class II, antigen-D-related (HLA-DR), present in a sample. 